\title{
En defensa de la persona y su agencia en el campo del neuromanagement. Una propuesta desde la filosofía de la acción de E. J. Lowe
}

\author{
In defense of the person and his agency in the field of neuromanagement. \\ A proposal from E. J. Lowe's philosophy of action
}

\author{
Gonzalo Flores-Castro Lingán ${ }^{1}$ \\ Universidad Católica San Pablo, Arequipa, Perú• gaflores@ucsp.edu.pe
}

\begin{abstract}
Resumen
El presente artículo muestra cómo la neurociencia aplicada al management asume ciertos supuestos antropológicos que reducen a la persona y su actuación a meras causas por eventos físicos (especialmente neuronales). Además de las críticas y advertencias, que los propios investigadores sostienen, se pretende mostrar no solo los problemas intrínsecos de tales supuestos, a saber, la pérdida del agente personal, sino también la imposibilidad de dejar de lado a la persona para explicar su actuación. Se expone, por tanto, la teoría estándar de la acción personal, que explica la acción en términos de causalidad por eventos físicos, mostrando algunos de sus problemas internos. Luego se argumenta la necesidad de la agencia personal, en términos de la persona como «sustancia personal», desde la filosofía analítica de E. J. Lowe. Al final, concluimos que no solo la teoría estándar es intrínsecamente problemática (al perder a la persona), sino que no es posible hacer inteligible la acción personal solo apelando a eventos físicos.
\end{abstract}

Palabras clave: filosofía de la acción, E. J. Lowe, teoría estándar de la acción, neurociencias, neuromanagement, neuromarketing, filosofía de la mente, teoría de la acción, antropología filosófica.

\footnotetext{
${ }^{1}$ Esta publicación ha sido posible gracias a una subvención de la John Templeton Foundation. Las opiniones expresadas en esta publicación son las del autor y no reflejan necesariamente los puntos de vista de la John Templeton Foundation. This publication was made possible through the support of a grant from the John Templeton Foundation. The opinions expressed in this publication are those of the author and do not necessarily reflect the views of the John Templeton Foundation.
}

illustro • Universidad Católica San Pablo, Arequipa • Vol. 12, 2021, 27-41 • e-ISSN 2710-2440 


\begin{abstract}
This article shows how neuroscience applied to management assumes certain anthropological assumptions that reduce the person and his agency to mere causes by physical (especially neural) events. In addition to the criticisms and warnings that the researchers themselves sustain, this paper intended to show not only the intrinsic problems of such assumptions, namely the loss of the personal agency, but also the impossibility of leaving aside the person to explain his agency. The standard theory of personal action, which explains action in terms of causation by physical events is exposed showing some of its internal problems. We then argue for the necessity of personal agency, in terms of the person as «personal substances», from the analytic philosophy of E. J. Lowe. In the end, we conclude that not only is the standard theory intrinsically problematic (by losing the person), but that it is not possible to make personal action intelligible only by appealing to physical events.
\end{abstract}

Keywords: philosophy of action, E. J. Lowe, standard theory of action, neurosciences, neuromanagement, neuromarketing, philosophy of mind, action theory, philosophical anthropology.

\title{
1. Introducción
}

Es evidente que las neurociencias están de moda, las podemos ver en campos tan diversos como el derecho (Sapolsky, 2017), hasta en dietas (Chaudhary, 2019) y fútbol (Crespo, 2019). El management no ha sido la excepción y existe, desde hace algunos años, un creciente interés en la aplicación de teorías y herramientas neurocientíficas para comprender y mejorar el comportamiento del consumidor (Ariely y Berns, 2010; Venkatraman et al., 2012), el comportamiento organizacional (Chattopadhyay, 2020; Waldman et al., 2016), el management general (Sulkowski y Chmielecki, 2017; Lindebaum, 2016) y hasta la ética organizacional (Dubljevic et al., 2020). Para comprender el alcance del fenómeno basta con revisar la ingente cantidad de literatura de difusión en management con el término neuro: por ejemplo, una búsqueda sencilla en Amazon.com muestra que hay más de mil títulos con los términos neuromarketing y neuromanagement.
Nadie duda de que las neurociencias tienen mucho que aportar (y aportan) a la comprensión de las empresas y los mercados. Como afirma Sulkowski y Chmielecki (2017), «la metodología de investigación neurocientífica ya ha proporcionado conocimientos bastante importantes sobre la gestión y, especialmente, el liderazgo, la innovación, la gestión del cambio y la toma de decisiones» (p. 61). Sin embargo, actualmente existe una brecha entre las investigaciones neurocientíficas que prometen una «mejora» del ser humano y los resultados que estas tecnologías puedan tener en el mundo real, tanto a nivel psicológico como sociológico (Racine et al., 2021). Lo cual no impide que intrépidos científicos y empresarios intenten aplicar dichos conocimientos a la práctica, asumiendo ciertos supuestos antropológicos para llenar algunos vacíos conceptuales a la hora de interpretar o entender las limitaciones de ciertos estudios. Veamos un par de casos. 
En el campo del neuromarketing se pretende utilizar la metodología propia de la investigación neurocientífica para «analizar, comprender y predecir el comportamiento humano con relación al mercado y al consumo de bienes de uso» (Manes y Niro, 2015, p. 176). Las empresas que lo utilizan «prometen brindarles a sus clientes la verdad acerca de lo que los consumidores piensan y sienten acerca de un producto» (ibidem, cursivas en el original). Esto supone un tipo de identidad entre nuestro conocimiento del comportamiento neuronal y el conocimiento de la mente del consumidor, es decir, asume que se puede explicar el comportamiento humano a partir del comportamiento neuronal. Bajo este supuesto, una empresa de neuromarketing (MindSign Neuromarketing) realizó un estudio en 2011 donde, a través de técnicas de fMRI, mostró que cierta área cerebral (la corteza insular) se activaba ante el sonido del celular. Dado que en estudios neurocientíficos anteriores dicha área cerebral se relacionaba con el sentimiento de amor, se concluyó que las personas literalmente amaban a sus iPhones, lo cual explicaría ciertos comportamientos compulsivos y adictivos de sus usuarios (Lindstrom, 2011).

Russ Puldrack, profesor de psicología y neurobiología de la Universidad de Stanford, resumió los resultados del estudio de la siguiente forma: «complete crap» (Puldrack, 2011); y Manes y Niro (2015) afirman que este estudio «evidencia los problemas que surgen cuando no se tienen en cuenta las limitaciones de una tecnología» (p. 177). En el fondo, estos resultados muestran lo poco de científico que tienen estos intentos de aplicación, pues, además de ser estudios que no cuentan con contraste de pares, sus conclusiones no explican «cuáles son sus limitaciones y cómo tienen que ser interpretadas sus conclusiones» (ibidem, p. 178). Al final, es el prestigio de la ciencia que se utiliza «como marketing del neuromarketing» (ibidem, $\mathrm{p}$. 177, cursivas en el original).

Por otra parte, en el campo del comportamiento organizacional existen diversas investigaciones que parten de un mismo supuesto antropológico, a saber, «que el comportamiento en entornos organizativos puede reducirse esencialmente a las neuronas de los cerebros de sus miembros» (Waldman et al., 2017, p. 439). Por ejemplo, en estudios realizados sobre la hormona oxitocina se observó que su administración intranasal eleva el nivel de confianza grupal (Van IJzendoorn y BakermansKranenburg, 2012). Aunque estos estudios han sido criticados (Nave et al., 2015), no ha dejado de tener interés por parte del mundo empresarial, dado que tiene utilidad «para las criaturas sociales que tratan de trabajar juntas» (Zak, 2017, p. 5). De hecho, se plantea sus posibles usos para la mejora de la confianza y rendimiento en equipos de trabajo (de Visser et al., 2017). Así, sería posible crear «culturas de confianza» «a través de [...] prácticas clave que estimulan la producción de oxitocina, una sustancia química que facilita el trabajo en equipo» (Zak, 2017, p. 1, cursivas nuestras). El lector avisado sabrá reconocer que las prácticas que se promueven (como el crecimiento de las personas o su reconocimiento) no se buscan por sí mismas, sino que son instrumentalizadas para obtener una elevación de oxitocina. $\mathrm{O}$, en otras palabras, lo que se busca es que, a través de estas prácticas, una persona se «dope». Parafraseando a Sinek: Vuélvase adicto a la oxitocina, ¡se lo ha ganado! (Sinek, 2017).

Se puede ver que la brecha entre neurociencias y aplicaciones prácticas, en entornos empresariales, es completada por una serie de supuestos antropológicos implícitos en la 
interpretación de los resultados neurocientíficos. Este supuesto, si es que no queda lo suficientemente claro, es una falsa o al menos problemática identificación de las causas de la acción personal con la causalidad "física» del mundo natural, especialmente con la correspondiente a los movimientos neuronales. Como ve claramente Robertson (2020, p. 124, cursivas nuestras):

Como metodología descriptiva, los resultados de la fMRI proporcionan correlaciones con un estado psicológico que se asocia con algún nivel de actividad neuronal elevada. Los cambios resultantes en el campo magnético medidos por la fMRI se utilizan como una aproximación a las áreas cerebrales que están activas durante una tarea o como respuesta a un estímulo [...]. [...] las críticas a los hallazgos de la neurociencia sostienen que existe una tendencia a la sobreinterpretación de los resultados. La afirmación es que las explicaciones de los estados mentales se extrapolan a partir de las descripciones de la actividad neuronal y que la correlación puede confundirse con la causalidad [...]. A pesar de que Churchland sostiene que los hallazgos de la fMRI no proporcionan explicaciones, Kable (2011) sostiene que se pueden hacer inferencias causales en el sentido de que los cambios en el entorno o en los estados psicológicos provocan cambios en la actividad cerebral. Este conjunto de argumentos sugiere que debemos seguir buscando formas en las que la neurociencia pueda ser útil para explicar la toma de decisiones y el comportamiento éticos, pero al mismo tiempo, debemos ser cautos a la hora de atribuir poderes explicativos a los hallazgos de la neurociencia.
Existe, pues, el peligro de un reduccionismo mecanicista en lo que respecta a la visión del hombre en el seno de una organización. Esta imagen de la persona «supone un cambio radical de perspectiva en las ciencias sociales y lleva a que el hombre sea destronado por la ciencia una vez más» (Sulkowski y Chmielecki, 2017, p. 52), pues implica un cambio en la ontología de la persona. Como mencionan Ackroyd y Fleetwood (2005, p. 11):

[...] todo el mundo tiene una ontología. Como científicos sociales, asumimos compromisos ontológicos o tenemos presupuestos ontológicos, aunque estos presupuestos permanezcan, por lo general, implícitos y sin examinar. Esto puede ser problemático porque la forma en que suponemos (implícita o explícitamente) cómo es el mundo social tiene una gran influencia no sólo en nuestra forma de estudiarlo (nuestra epistemología), sino en la forma de conceptualizar todo el marco analítico que adoptamos.

Es como si, en el fondo, no hubiésemos abandonado del todo la administración científica tayloriana. Lo cual es fácil de entender, pues la administración científica no solo era una visión mecanicista de la empresa, sino que era una visión acerca del ser humano y las organizaciones en general, una cosmovisión, una filosofía (Martínez-Echevarría, 2001) y hasta, para Stewart (2009, p. 65), una religión:

Taylor did not invent a machine, but a religion —one that remains exactly where Taylor left it. Management theory today, as the technological rhetoric of gurus like Hamel and Drucker makes clear, answers to the same hopes and contradictions that gave rise to scientific management, and 
so it continues to worship at the feet of the idol Taylor created.

Por supuesto, estas críticas están presentes en diversos investigadores. Con relación a la teoría de la organización y gestión, por ejemplo, Waldman, Ward y Becker (2017, p. 439, énfasis nuestro) sostienen que:

[...] aunque las primeras neurociencias básicas podían interpretarse de este modo [identificando comportamiento y causalidad neuronal], [...] las primeras piezas conceptuales o de pensamiento de la [...] $\mathrm{ON}$ [Organizational Neuroscience] advertían que las tecnologías existentes no eran capaces de reducir el comportamiento al nivel de las neuronas [...]. Además, Waldman et al. (2015a) advirtieron a los investigadores de la $\mathrm{ON}$ que no debian incurrir en un reduccionismo excesivo. Su advertencia se inspiró en el trabajo de Lindebaum y sus colegas (Lindebaum \& Jordan 2014, Lindebaum \& Zundel 2013) que planteaba dudas sobre hasta qué punto los aspectos complejos de la organización podían reducirse a las neuronas o regiones cerebrales de los miembros individuales de la organización. Lindebaum \& Zundel (2013, p. 871), sin embargo, también afirmaron que «el cerebro desempeña incuestionablemente un papel importante... [aunque] puede no ser siempre el punto de partida que desencadena el comportamiento humano». Por el contrario, aunque el comportamiento tiene ciertamente su origen en redes neuronales específicas, pasarán muchos años antes de que los investigadores puedan comprender toda la complejidad del cerebro humano. Por supuesto, esto no quita la utilidad de explorar las regiones y redes del cerebro para avanzar en la teoría de la organización y la gestión.

Este tipo de reduccionismo no solo se encuentra en la empresa, sino en todo el pensamiento económico de base neoclásica (MartínezEchevarría, 2015). En palabras de Pilkington (2016): «Los economistas de hoy en día se adhieren instintivamente a una especie de vulgar visión newtoniana del mundo. Es decir, instintivamente piensan en términos de un espacio en el que se desenvuelven una serie de fuerzas» (p. 345). Y es algo bastante discutido en la filosofía de la mente y de la acción. Por ejemplo, para el filósofo, médico y neurocirujano clínico Raymond Tallis, en la actualidad existe una cierta neuromanía, «la apelación al cerebro, tal como es revelado a través de la ciencia más reciente, para explicar nuestro comportamiento» (Tallis, 2011, p. 5). Tallis sostiene que los neuromaniáticos tienen dos grandes errores: «el primero es técnico — las limitaciones del fMRI, el diseño de los estudios que lo utilizan y la manera en que la data es analizada- [...] [el segundo y] mucho más importante, son las fallas que nacen de errores conceptuales [filosóficos]» (Tallis, 2011, p. 76).

En el presente artículo abordaremos estas cuestiones conceptuales - filosóficas - de fondo, que nos permitirán entender el problema de asumir un tipo de reduccionismo de identificación de las causas de la acción personal con la causalidad «física» del mundo natural. Partiendo de la filosofía de la acción se analizará el por qué realizar dicho reduccionismo resulta, en última instancia, en la pérdida del agente personal y, por tanto, de su libertad. Para ello, revisaremos la propuesta estándar de la teoría causal de la acción humana en términos de eventos físicos, tratando de hacer notar sus deficiencias. Más aún, intentaremos 
hacer una formulación de argumentos desde la propuesta de E. J. Lowe, quien fue uno de los grandes críticos de dicha teoría. Para lograrlo dividiremos este artículo en dos partes: la primera buscará una breve descripción de la propuesta estándar, así como una breve crítica hacia algunos de sus puntos; en la segunda propondremos una argumentación inspirada en E. J. Lowe, para develar la insuficiencia teórica de la propuesta causal física.

\section{Causalidad por eventos físicos}

La acción personal es un tema frecuente en distintas disciplinas que estudian al ser humano. Entre los diversos temas que se tratan se encuentra la cuestión de la agencia. En términos muy generales, un agente es aquella entidad que tiene la capacidad de actuar, mientras la «agencia» «denota el ejercicio o manifestación de dicha capacidad» (Schlosser, 2019). En el caso del ser humano, agencia sería la capacidad que tiene la persona humana de actuar, de ejercer una acción o, en otros términos, de «actuar» más que «ser actuado».

Para explicar la agencia personal se suele recurrir a la teoría de la acción «estándar», la cual suele explicar la intencionalidad propia de los actos humanos en términos de causalidad por eventos físicos o a través de estados mentales (o ambos). Aunque la teoría de la acción desde un punto de vista de causalidad por eventos físicos cuenta con bastante aceptación entre filósofos profesionales, se ha argumentado de diferentes formas el por qué esta teoría de la acción no solo es insuficiente, sino que falla al querer explicar la agencia, especialmente en relación con la agencia humana (Schlosser, 2019). En esta primera sección se expondrá la explicación de la agencia desde la causalidad por eventos físicos, es decir, a través de la reducción del comportamiento humano a sus causas físicas (biológicas, cerebrales, neuronales, etc.).

El principio básico común de todas las teorías causales de la acción es que «el agente realiza una acción sólo si un apropiado estado interno del agente provoca, en cierto sentido, un resultado particular» (Davis, 2010, p. 32). En otros términos, algo en el agente es la causa de la acción, no es solo actuado, sino que de alguna manera actúa. Desde una perspectiva análoga se puede aplicar este principio tanto a realidades inanimadas, como un árbol, o animales superiores, tales como primates, o seres racionales que suponemos libres, como el ser humano.

Ahora, si bien este principio es aplicable a distintas realidades, en la teoría de la acción se suele distinguir entre seres inertes y realidades que, en cierto sentido, provocan una acción, como los vivientes en general y los humanos en particular. Es importante notar esto, pues lo que diferencia una cosa de otra es que hay cierta novedad en la agencia, «aporta algo», pudiendo la causa ser la complejidad del organismo del viviente, cuya carga genética y mecanismos psicológicos e instintivos explican la acción o, en el caso del ser humano, es su volición la que explica su agencia. De hecho, desde Aristóteles el estudio de la agencia se centra más en este último: «el hombre es el único que es principio de ciertas acciones [...] no podemos decir de ningún otro animal que actúa» (Ética a Eudemo, 2, 6, 1222 b 20).

La distinción tiene importancia porque, a diferencia de la actividad del mundo inorgánico estudiada desde una perspectiva de acción física, la agencia humana utiliza otros términos como libertad, intencionalidad, razones o motivos, que compiten en la explicación causal de la acción humana. La teoría estándar busca, 
como mencionamos, dar una explicación causal por eventos físicos de aquello que se pretende explicar por aquellos términos (libertad, intencionalidad, etc.). En ese sentido, la versión más defendida por filósofos profesionales es el compatibilismo, que «alude a la compatibilidad entre una descripción científica determinista (o, al menos, impersonal) subyacente y un vocabulario macroscópico de elección y volición» (Carroll, 2017, p. 422).

Desde una perspectiva naturalista se suele argumentar que hablar de nociones como libertad e intencionalidad en la acción humana, tomadas como «realidades» con efectos causales en el mundo físico, viola el principio del cierre causal del mundo físico. Así pues, si bien no es posible, en este momento, conocer con exactitud la explicación física de la agencia humana, es decir, no contamos con un modelo matemático que nos permita entender el rol que juega el sistema físico humano para explicar la acción humana, eso no quiere decir que la explicación escape a un conjunto de eventos físicos. Así, si se nos proporcionase el estado cuántico completo y exacto correspondiente a «una persona que elige estudiar derecho», y disponemos de las capacidades de cálculo del Demonio de Laplace, podríamos predecir con precisión la acción de la persona que elige "estudiar derecho», no requiriendo de más información para explicar su agencia (Carroll, 2017).

Ahora bien, una explicación causal a partir de eventos físicos no es un análisis semántico. Ninguna definición a partir de un modelo matemático o descrito en términos de causalidad física sería sinónimo de «el agente $A$ causó $e$ intencionadamente». Sin embargo, «el fracaso de esa teoría causal como análisis semántico no socava su éxito a la hora de arrojar luz sobre lo que es actuar intencionadamente» (Davis, 2010, p. 33). La idea básica es que, si el agente hace algo intencionadamente, y si sus intenciones son eficaces, entonces - dado el cierre causal del mundo físico - algún evento o conjunto de eventos físicos están detrás de la acción ejerciendo su poder causal.

Esto lleva a los teóricos causales a proponer, al menos, dos salidas. La primera suele entender los fenómenos mentales, la intencionalidad, la libertad o incluso el propio agente personal («yo» o persona) como epifenómenos del mundo físico. Así, por ejemplo, Fuster (2019) considera que los eventos físicos que ocurren en la actividad cortical del cerebro son lo que explican lo que él llama «ciclo percepción/ acción», siendo la conciencia, o el pensamiento deliberado, epifenómenos de la actividad cerebral, no haciendo falta recurrir a un «centro de voluntad» o a un «ejecutivo central» para explicar la agencia humana, especialmente la libertad. Otra forma de proponer esto es hablando de un reduccionismo fuerte, es decir, afirmar que la intencionalidad, la libertad o la agencia personal no es más que causalidad física. Así, una afirmación del tipo «el agente $A$ causó el evento $e$ » solo es posible si «el agente $A$ causó el evento $e$ si y sólo si hubo algún evento [físico], $x$, tal que $x$ involucró a $A$ y $x$ causó $e$ » (Lowe, 2008, p. 123).

La segunda, y más difundida entre filósofos profesionales contemporáneos, es aquella que intenta compatibilizar el vocabulario intencional o de agencia personal con el propio del lenguaje de ciencias físicas, pero teniendo cuidado de no mezclar los tipos de semántica. Así, es posible «describir lo que está ocurriendo en términos de señales electroquímicas [...], o en términos de [...] estados mentales [...]. Pero hay que tener cuidado de no equivocarse, 
y empezar una frase en un idioma y tratar de terminarla en otro» (Carroll, 2017, p. 417). Los estados mentales o incluso las personas son solo formas de hablar de estados físicos particulares, sin embargo, ya que no tenemos la capacidad de cálculo del Demonio de Laplace ni conocemos nuestro propio estado cuántico (o del universo, o nuestros estados neuronales, etc.), no podemos más que usar el lenguaje intencional al hablar de nuestra propia agencia. Ambos vocabularios son legítimos. Sin embargo, decir que uno toma decisiones libremente y afirmar que esa «decisión libre» introduce algo al sistema físico, sería violar las leyes naturales, superando las leyes de la física a través del pensamiento o de una sustancia personal no identificable con ningún átomo. Evidentemente esto implica una subordinación de lo intencional a la causalidad física, aunque respetando su autonomía semántica. Lo importante es que, aun si existiese una realidad emergente con propiedades que solo pueden ser descritas con lenguaje de estados mentales o sustancias metafisicas, no existe una causalidad descendente de la intencionalidad, la volición o el «yo» hacia el mundo físico, dado el cierre causal del mundo físico.

Ambas versiones no dejan de tener problemas, así como el argumento de fondo, centrado en el cierre causal del mundo físico. Entre las varias objeciones que se pueden presentar, podríamos decir que, apelar al cierre causal del universo físico es precisamente asumir lo que se intenta probar: que la causalidad por eventos físicos puede explicar la agencia humana, ¿qué sucede si no puede? La segunda versión apela al Demonio de Laplace, asumiendo en cierta medida que, cuando la física esté completa, tendremos la información de todos los posibles eventos físicos, con lo que será posible predecir la agencia. Esto no deja de ser, en ambos casos, una apelación a la ignorancia. No sabemos si tendremos algún día un banco de información de todo evento físico, así como no sabemos si tendremos la matemática adecuada para interpretarlos. Evidentemente esto no prueba la existencia de la libertad o la intervención de una sustancia racional, pero los argumentos brindados por quienes sostienen un reduccionismo de la agencia humana tampoco prueban su contrario. Una causa distinta a la que estudia la física podría explicar la agencia personal; que esto sea irrelevante desde la perspectiva física no es razón para desestimar otros planos de la realidad que afecten a la realidad misma de la agencia personal. Afirmar lo contrario es reducir la realidad al método. Asumir el cierre causal físico en la agencia humana es tomar las causas físicas con un peso ontológico que por sí mismas no poseen (Sanguineti, 2007).

Por otra parte, no tenemos una clara definición de lo que una acción física es (Arana, 2015). «El hecho de que los libros de física y química solo se ocupen de acciones físicas no implica que todas las acciones del mundo físico lo sean. Eso sería tanto como afirmar que no queda en el universo ningún misterio por explorar» (Arana, 2015, p. 42). En palabras de Bertrand Russell (1985): «No siempre se cae en la cuenta de lo [...] abstracta que resulta ser la información proporcionada por la física [...]. Establece ciertas ecuaciones fundamentales que permite lidiar con la estructura lógica de los fenómenos, dejando completamente en la oscuridad el carácter intrínseco de los fenómenos [...]» (p. 13). Evidentemente estas objeciones tocan aspectos que escapan a la misma teoría causal de la acción, entrando a una crítica acerca de la metafísica general del mundo natural. Es por ello que, en el siguiente apartado, sin entrar en la discusión sobre la metafísica de la realidad como un todo, argumentaremos 
sobre la necesidad de las causas mentales, así como la causalidad agente como formas de entender la agencia humana que superan la teoría estándar. Esto lo haremos, como se mencionó, tomando como base al pensamiento de E. J. Lowe (2006, 2008).

\section{La irreductibilidad de la causalidad sustancial en la agencia personal}

La propuesta de la teoría causal estándar es, evidentemente, una propuesta naturalista y reduccionista. De hecho, en filosofía de la acción se pretende a veces invocar una irreductible causalidad agente, sin embargo, es rechazada por ser incompatible con la ortodoxia naturalista en filosofía de la mente de corte analítico, la cual supone que toda causalidad es causalidad por eventos (Lowe, 2008) (entendidos los eventos como eventos físicos). Siguiendo a E. J. Lowe, presentaremos dos argumentos que permiten criticar la posición estándar, en primer lugar, mostrando cómo la agencia personal (que entenderemos como causalidad sustancial) no puede ser reducida a la causalidad por eventos; es más, solo es inteligible dicha reducción si la inversa es cierta, a saber, que la causalidad por eventos solo se entiende por causalidad sustancial. El segundo argumento pretende mostrar cómo hablar de dos interpretaciones semánticas distintas no resuelve el problema de la agencia personal; es más, por una parte, se pierde al agente actuante $y$, por otra, no es posible entender la agencia a partir de eventos físicos sin recurrir a nociones semánticas de otra índole.

Lo primero que hay que aclarar es que no necesariamente existe un tipo de causalidad. Es decir, el análisis que se pretender realizar no descarta que exista o se pueda explicar cierta causalidad a partir de eventos físicos.
Al contrario, se asume que pueden existir distintos tipos de causalidades. Desde esta perspectiva, podríamos empezar analizado el lenguaje que se utiliza para referirnos a la causalidad (no porque la causalidad se reduzca al lenguaje, sino porque el análisis lingüístico nos puede dar luces acerca de los supuestos metafísicos). Así, podemos tener la siguiente proposición: «el movimiento del brazo causó la ruptura del jarrón». En este caso, el verbo transitivo causar relaciona dos eventos físicos, el movimiento del brazo y la ruptura del jarrón. Pero también podemos expresarnos de la siguiente forma: «Juan Antonio causó la ruptura del jarrón». En este caso, el verbo causar relaciona una sustancia individual (un objeto particular persistente, en este caso una persona) con un evento físico, la ruptura del jarrón (Lowe, 2008).

Desde una perspectiva de causalidad por eventos físicos, la segunda forma de expresión no es más que una forma abreviada de una proposición más compleja que relacione eventos físicos, algo como «la sustancia $\mathrm{S}$ (Juan Antonio) causó el evento e2 si y sólo si hubo algún evento, e1, tal que e1 involucró a S y e1 causó e2» (I). Dicho de otra forma, el defensor de la teoría causal estándar trataría de mostrar que hablar de «Juan Antonio» como un «ejecutivo central» $\mathrm{o}$ «centro de voluntad» no es más que un epifenómeno, pudiendo ser reducido a mera causalidad por eventos físicos. Sin embargo, para que la reducción se dé efectivamente, tendrá que especificar qué quiere decir que una sustancia individual, es decir, Juan Antonio, esté involucrado en un evento.

Evidentemente, que el evento e involucre a la sustancia $\mathrm{S}$ no puede ser una simple yuxtaposición espacio temporal entre e y S. Esto se debe a que, en todo caso, podría haber ocurrido 
el evento «movimiento telúrico», y haber ocurrido también el evento «ruptura de jarrón», y Juan Antonio haber estado allí, inerte e inmutable. El proponente de la teoría causal por eventos físicos tiene en mente el evento que causa, el movimiento del brazo, como la explicación suficiente de la ruptura del jarrón, es decir, que la noción de involucramiento debe permitir decir que la sustancia individual Juan Antonio estuvo involucrada en la ruptura del jarrón, de una forma que permita distinguirla de un movimiento telúrico o cualquier otro evento físico. De no ser el caso, en realidad Juan Antonio no actuó, pues el evento físico podría haberse debido a otros eventos absolutamente impersonales (recordar la distinción que se hizo entre agencia humana y mundo inorgánico).

Este último problema se puede ver desde el siguiente ángulo: los científicos, como Fuster o Carroll, son una especie de sustancias que diseñan y hacen observaciones científicas, sacando sus conclusiones en sus respectivos campos, etc. De no ser una especie de sustancia la que actúe, sino que su actividad es una serie de eventos físicos, entonces, puesto que los eventos de deducir una teoría, hacer una determinada observación o realizar una determinada predicción matemática son eventos distintos, parece ser que no hay una cosa, la persona, la que lleve a cabo estas actividades (en corto, no hay agencia) y, por tanto, no se puede decir que los científicos sean quienes proponen la teoría que intenta explicar su propia agencia (científica). En ese sentido, de no involucrar la sustancia de alguna manera, la teoría parece ser autodestructiva (Feser, 2014).

El teórico causal por eventos físicos podría replicar que, en realidad, la persona es un conjunto de elementos físicos (por complejos que sean, en la corteza prefrontal o cualquier lugar, como plantea Fuster). El problema de recurrir a esta salida proviene del hecho de que ningún elemento en el conjunto implica necesariamente al sujeto actuante, con lo que, a fin de cuentas, se sigue perdiendo la agencia personal. Por ejemplo, el evento «pronunciar un discurso», ¿a qué conjunto de relaciones de eventos físicos (neuronales, cuánticos, etc.) pertenece?, ¿al conjunto que proporciona identidad a «Juan Antonio» o al que le da identidad a «Nancy Elizabeth»? Nada en el evento en cuestión involucra a una u otra sustancia, por lo que el evento «pronunciar un discurso», ¿acción de quién es? (cfr. Oderberg, 2007, pp. 77-78). Nuevamente, se pierde la agencia personal.

Entonces, se mantiene en pie que, de alguna manera, la causalidad por eventos físicos debe involucrar a la sustancia para salvar la agencia, es decir, algo como (I). ¿Cómo se debe entender este involucramiento? De acuerdo con Lowe (2008), podemos pensar que «El evento e1 causó el evento e2 
sólo en el caso de que hubiera alguna sustancia, $\mathrm{s} 1$, y alguna sustancia, s2, y alguna manera de actuar, F, y alguna manera de actuar, G, tales que e1 consistiera en la realización de $\mathrm{F}$ de $\mathrm{s} 1$ y e2 consistiera en la realización de $\mathrm{G}$ de $\mathrm{s} 2$, y s1, por F, causara el G de s2» (II) (Lowe, 2008, p. 145). De ese modo, podemos afirmar a la manera de (II) que «el movimiento del brazo de Juan Antonio causó la ruptura del jarrón», porque suponemos que fue la sustancia Juan Antonio la que, al mover su brazo, causó la ruptura del jarrón. Así, para que una sustancia esté involucrada en un evento, en el sentido de (I), basta con que ese evento «consista en que esa sustancia actúe de una determinada manera» (Lowe, 2008, p. 145).

De ser esto así, la forma (I) queda reducida a esto: "La sustancia $\mathrm{S}$ causó el evento e2 sólo en el caso de que hubiera algún evento, e1, y algún modo de actuar, F, tal que e1 consistiera en la realización del $\mathrm{F}$ de $\mathrm{S}$, y e1 causara e2» (I*) (Lowe, 2008, p. 146). El problema es que, si (II) es correcto, entonces «e1 causó e2» solo es verdad si existe «alguna sustancia, S1, y alguna manera de actuar, F, tal que e 1 consistía en la realización de $\mathrm{F}$ de $\mathrm{S} 1$, y S1, por la realización de F, causó e2». Así, en el supuesto de que (II) sea verdadera, (I*) es equivalente a: «La sustancia $\mathrm{S}$ causó el evento e 2 sólo en el caso de que hubiera algún evento, e1, y algún modo de actuar, F, tal que e 1 consistiera en la realización de $\mathrm{F}$ de $\mathrm{S}$, y S, por la realización de F, causara e2» (I**) (Lowe, 2008, p. 146). ¿Qué quiere decir esto? Pues que afirmar que «siempre que una sustancia causa un evento, lo hace actuando de una determinada manera y que su actuación de tal manera constituye un evento» (ibidem, p. 147). La implicación es que (I) es verdadera porque es equivalente a una causalidad sustancial $\left(\mathrm{I}^{* *}\right)$, y, por lo tanto, en vez de poder reducir la causalidad sustancial a una causalidad por eventos físicos, en realidad es la causalidad por eventos la que es reductible a una causalidad sustancial (ibidem).

Por otra parte, desde la perspectiva que expone Carroll (2017), una forma semántica no puede ser reducida a otra, es decir, la explicación en términos de agencia personal, intenciones, etc., no puede reducirse a términos de eventos físicos. No son dos explicaciones en competencia, sino explicaciones distintas de la agencia humana. Sin embargo, al ser la realidad fundamental el mundo físico, y dado el cierre causal del mundo físico, la explicación por agentes personales (sustancias), intenciones, razones, etc., no puede contradecir a la explicación por eventos físicos. Es más, el hecho de que la utilicemos solo muestra que nuestra capacidad cognoscitiva es limitada, pues si fuésemos el Demonio de Laplace y conociéramos con certeza nuestro estado cuántico, podríamos predecir con precisión la acción que se realizará.

Pues bien, la crítica que hemos realizado también es aplicable en este caso, porque la explicación por eventos físicos de alguna manera debe implicar la agencia personal (la sustancia actuante) para que se pueda considerar agencia. La salida de la distinción semántica, aunque plausible para no caer en traspaso epistémico (Ballantyne, 2018), no permite resolver el problema que, desde la perspectiva de la causalidad por eventos físicos, se pierde el agente. Esto se ve especialmente si se considera que la explicación a ese nivel epistémico (físico, neuronal, etc.) es la fundamental y que el nivel del agente (sustancia, con sus intenciones, voliciones, etc.) no tiene una influencia causal sobre el nivel fundamental.

Pero, además de dicha perspectiva, se puede argumentar desde otro punto de vista. Siguiendo 
a Lowe (2006), podemos proponer que las condiciones de identidad de la persona con su cuerpo difieren radicalmente: por ejemplo, es perfectamente concebible que una persona pueda ir cambiando cada célula de su cuerpo por una parte biónica, hasta reemplazar todo su cuerpo y aún ser esa persona, por lo que la persona puede ser distinta de su cuerpo o alguna parte del cuerpo y, por tanto, es distinta del cuerpo. De ser esto así, ninguna relación causal de eventos físicos podrá identificarse con la persona, ni la involucraría de alguna manera que sea distinta a (II) (con las respectivas consecuencias). Por lo que, si no es posible identificar a la persona con algún evento físico o conjunto de eventos físicos, la persona queda fuera de la ecuación $\mathrm{y}$, por tanto, nuevamente se pierde la agencia personal. Por otra parte, para Lowe (2006) es imposible identificar la causalidad mental con la causalidad física, así, por ejemplo, el acto mental de decisión — causalidad mental: singular, unitaria y distinta - no puede identificarse con ningún evento del sistema nervioso o cerebro, cuya causalidad física se puede ramificar como un árbol, no presentando un solo foco causal identificable y distinto (cfr. Penrose, 2015). Así, en este caso también las condiciones de identidad del tipo de causalidad son distintas.

Por otra parte, la causalidad mental es intencional, es decir, la causa se dirige hacia cierto tipo de efectos; sin embargo, la causalidad física no es de este tipo, es ciega, las causas físicas no se dirigen hacia ningún tipo o rango de efectos (cfr. Tallis, 2011; Lowe, 2006). Ambos tipos de causalidad se requieren para explicar una acción: puedo explicar el movimiento de mi brazo por medio de una decisión (causalidad intencional) y por los movimientos de músculos, nervios y neuronas (causalidad física). Esta distinción es fundamental, porque la causalidad física, al ser no-intencional, solo explica eventos no hechos; es decir, puede explicar una serie de eventos neuronales, los cuales en sí mismos no explican el hecho de un movimiento en particular. Así, si bien podemos afirmar con Carroll (2017) que la distinción semántica es necesaria y es mejor no confundir, también es cierto que se requiere de ambas explicaciones causales para no perder la agencia personal. Por ejemplo, se podría explicar los eventos físicos de que un pulmón conserve el oxígeno inhalado, pero no podría explicar si es que este evento fue intencional o no, podría ser solo un evento casual, una coincidencia, o algo buscado - intencional-, como en el hecho particular y concreto de un buzo sumergiéndose en el mar (en última instancia, si fue una acción de un agente, si hubo agencia o no). Ambas explicaciones son distintas e irreducibles la una a la otra; sin embargo, no implican separabilidad o irreductibilidad, ni mucho menos que una sea más fundamental que otra.

\section{Conclusión}

El presente artículo ha mostrado los reduccionismos antropológicos que se cometen en el neuromanagement, especialmente a la hora de interpretar los resultados. En el fondo, se parte del supuesto de que el comportamiento humano es explicable en términos de causas físicas (especialmente cerebrales o neuronales). Esto no es solo algo del management sino de la teoría económica neoclásica en general. La idea rectora es que la acción personal es explicable en términos de eventos físicos (sean los que fueren), perdiendo inadvertidamente al agente que actúa: la sustancia personal. Esto es, en última instancia, un retorno al taylorismo.

Sin embargo, no nos hemos limitado a hacer notar las falencias o críticas al neuromanagement. Hemos intentado demostrar conceptual y filosóficamente que la antropología que se asume, la 
teoría estándar de la acción personal en términos de causalidad por eventos físicos, además de los problemas intrínsecos que pueda tener (apelar a la ignorancia o la dificultad de definir lo que es una acción física), no puede explicar la agencia humana sin perderla en el proceso. Tratar de entender al agente personal (la sustancia personal) como un epifenómeno ilusorio, reducible a una explicación por eventos físicos, no deja de perder la agencia si deja de lado la forma en que los eventos físicos involucran de alguna manera al agente que actúa. Además, tratar de involucrar a la sustancia que actúa en la causalidad por eventos físicos termina jugando en contra de la teoría estándar, es decir, es también plausible que los eventos físicos sean los reducibles a la causalidad sustancial.

Por otra parte, apelar a la compatibilidad entre dos formas semánticas (y epistémicas) de explicar la agencia, teniendo como fundamental la causalidad del mundo físico (dado el naturalismo metafísico que se presupone), tampoco resuelve el problema, pues, ¿cómo involucrar al agente si ni siquiera es posible mezclar semánticas? Tratar de identificar a la persona con un evento o conjunto de eventos físicos tampoco parece resolver el problema, pues las condiciones de identidad son distintas en cada caso. Peor aún, la agencia personal se pierde si solo se apela a eventos físicos, que en sí mismos no indican algún tipo de acción (podrían ser accidentes, ser simplemente actuados de forma extrínseca), por lo que, si la realidad fundamental es la que se explica por causalidad por eventos físicos, hace ininteligible la acción personal.

Es así que, como hemos mostrado, es posible defender la agencia personal, la persona actuante (la sustancia personal), de los reduccionismos implícitos de las neurociencias aplicadas al management. No es pues reducible a una causalidad por eventos físicos sin perder, implícita y necesariamente, a la persona que actúa. Vale no solo la advertencia sino la demostración de las consecuencias de asumir dicho reduccionismo en todo el campo de la administración. Al estudioso del management interesado en la reflexión sobre temas neurocientíficos y filosóficos - especialmente antropológicos y de filosofía de la mente-, resulta un buen punto de partida las obras de Feser (2007), Sanguineti (2007, 2014) y Manes (2014, 2018); para una crítica de los límites de las neurociencias y la visión naturalista del ser humano y del mundo, es relevante la obra de Tallis (2011) y Pérez (2021), aunque ambas requieran un poco de formación filosófica.

\section{Referencias bibliográficas}

Arana, J. (2015). La conciencia inexplicada. Madrid: Biblioteca Nueva.

Ariely, D. y Berns, G. S. (2010). Neuromarketing: the hope and hype of neuroimaging in business. Nature Reviews Neuroscience, Abril; 11(4): 284-292.

Aristóteles. (1984). The Complete Works of Aristotle. Vol. II. Jonathan Barnes (ed.). Princeton University Press. West Sussex. 
Carroll, S. (2017). El Gran Cuadro. Los orígenes de la vida, su sentido y el universo entero. Pasado y Presente.

Chaudhary, K. (2017). Reprográmate: el (infalible) plan neuroayurvédico para perder peso, desintoxicarte y alcanzar la plenitud. Vintage Español.

Davis, W. A. (2010). The Causal Theory of Action. En T. O'Connor y C. Sandis, A Companion to the Philosophy of Action (pp. 32-39). West Sussex: Wiley-Blackwell.

De Visser, E. J., Monfort, S. S., Goodyear, K., Lu, L., O’Hara, M., Lee, M. R., ...y Krueger, F. (2017). A little anthropomorphism goes a long way: Effects of oxytocin on trust, compliance, and team performance with automated agents. Human factors, 59(1), 116-133.

Feser, E. (2007). Philosophy of mind. London. Oneworld Publications.

Feser, E. (2014). Scholastic Metaphysics. A contemporary introduction. Heusenstamm: Editions Scholasticae.

Fuster, J. M. (2019). Neurociencia. Los cimientos cerebrales de nuestra libertad. Ciudad de México: Paidós.

García, M. J. C. (2019). Neurociencia aplicada al fútbol. Wanceulen SL.

Lindstrom, M. (2011). You Love Your iPhone. Literally. The New York Times. Sept. 30. URL: https://www.nytimes. com/2011/10/01/opinion/you-love-your-iphone-literally.html

Lowe, E. J. (2006). Non-Cartesian Substance Dualism and the Problem of Mental Causation. Erkenntnis (1975-), 65(1), 5-23.

Lowe, E. J. (2008). Personal Agency. The Metaphysics of mind and action. New York: Oxford University Press.

Manes, F. y Niro, M. (2014). Usar el cerebro. Conocer nuestra mente para vivir mejor. Buenos Aires. Planeta.

Manes, F. y Niro, M. (2018). El cerebro del futuro. ¿Cambiará la vida moderna nuestra esencia? Buenos Aires. Planeta.

Martínez-Echevarría-y-Ortega, M. A. (2001). Teorías de la empresa y crisis de la modernidad. Cuadernos de Empresa y Humanismo, 83. Universidad de Navarra.

Martínez-Echevarría-y-Ortega, M. A. (2015). ¿Tiene sentido una teoría de la empresa? Cuadernos de Empresa y Humanismo, 128. Universidad de Navarra.

Nave, G., Camerer, C. y McCullough, M. (2015). Does oxytocin increase trust in humans? A critical review of research. Perspectives on Psychological Science, 10(6), 772-789.

Van IJzendoorn, M. H. y Bakermans-Kranenburg, M. J. (2012). A sniff of trust: meta-analysis of the effects of intranasal oxytocin administration on face recognition, trust to in-group, and trust to out-group. Psychoneuroendocrinology, 37(3), 438-443.

Oderberg, D. (2007). Real Essentialism. New York: Routledge.

Penrose, R. (2015). La nueva mente del emperador. 2.a ed. México D. F.: Fondo de Cultura Económica. 
Pérez, M. (2021). La cosmovisión naturalista. Consecuencias epistemológicas, ontológicas y antropológicas. Salamanca. San Esteban Editorial.

Pilkington, P. (2016). The Reformation in Economics. Palgrave Macmillan, Londres.

Poldrack, R. (2011). NYT Op-Ed + fMRI = complete crap. Entrada en blogspot. URL: http://www.russpoldrack. org/2011/10/nyt-editorial-fmri-complete-crap.html

Racine, E. Sattler, S., y Boehlen, W. (2021). Cognitive Enhancement: Unanswered Questions About Human Psychology and Social Behavior. Science and Engineering Ethics, 27(2), 1-25.

Robertson, D. C. (2020). Decision Neuroscience and Organizational Ethics. En Joé T. Martineau y Eric Racine (eds.), Organizational Neuroethics. Reflections on the Contributions of Neuroscience to Management Theories and Business Practices, Springer, Cham, pp. 109-130.

Russell, B. (1985). My Philosophical Development. London: Unwin Paperbacks.

Sanguineti, J. J. (2007). Filosofía de la mente. Madrid: Palabra.

Sanguineti, J. J. (2014). Neurociencia y filosofía del hombre. Madrid: Palabra.

Schlosser, M. (2019). Agency. (E. N. Zalta, Editor). Obtenido de The Stanford Encyclopedia of Philosophy: https:// plato.stanford.edu/archives/win2019/entries/agency

Sinek, S. (2017). Los líderes comen al final. 3.a ed. Ediciones Urano. Barcelona.

Sułkowski L. y Chmielecki, M. (2017) Application of Neuroscience in Management, en K. Nermend y M. Łatuszynska (eds.), Neuroeconomic and Behavioral Aspects of Decision Making, Springer Proceedings in Business and Economics.

Stewart, M. (2009). The management myth: Debunking modern business philosophy. WW Norton \& Company.

Tallis, R. (2011). Aping Mankind. Durham: Acumen.

Venkatraman, V., Clithero, J. A., Fitzsimons, G. J. y Huettel, S. A. (2012). New scanner data for brand. Journal of Consumer Psychology, 22(1), 143-153.

Waldman, D. A., Ward M. K. y Becker, W. J. (2017). Neuroscience in Organizational Behavior, Annual Review of Organizational Psychology and Organizational Behavior, (4), pp. 425-444.

Zak, P. J. (2017). The Neuroscience of Trust. Management behaviors that foster employee engagement. Harvard Business Review, January-February issue. 